\title{
Rapid Identification and Susceptibility Testing of Candida Species, from Urine by MALDI-TOF Mass Spectrometry and VITEK 2
}

\author{
Soha Abdallah Moursi \\ Clinical Laboratory Science, College of Applied Medical Sciences/Hail University, KSA \\ Department of Bacteriology, Mycology and Immunology, Faculty of Veterinary Medicine, Zagazig University, Egypt
}

\begin{abstract}
Urinary tract infection(UTI) specially with Candida Spp., are mostly common in females which are suffering from vaginitis or got the infection through usual gynecology clinic visits and as a nosocomial infection specially in case of using urinary catheterization . Early identification of Candida spp., is necessary especially in nosocomial UTI and it could help selecting appropriate antifungal for treatment. This study shows that Matrix-Assisted Laser Desorption/Ionization Time of Flight Mass Spectrometry( MALDI-TOF MS) is a reliable tool for fast and accurate identification of Candida spp.,. 146(4.3\%) positive urine samples for yeast infection out of 3,370 samples were submitted to Microbiology laboratory of Hail General Hospital and Hail Maternity Hospital from April 2014 to December 2015. Specimens were direct microscopically examined for fungal elements and cultured on Sabouraud's Dextrose agar (SDA), Candida Chromogenic agar and Blood agar as a routine work identification, isolates were analyzed using (MALDI-TOF MS) and Vitek 2 for identification and susceptibility testing .The results showed that more than $70 \%$ of the isolates were C.albicans ,10\% C.galabrata,8\%C. Kursi, 5\%C.dublinsiensis,4\% C. lusitaniae and 3\% C.tropicalis. In conclusion Vitek 2 susceptibility system AST-YSO7 cards and MALDI-TOF MS can be used for accurate routine clinical identification. Comparing to the Vitek 2 , MALDI-TOF MS is easier, faster and more economic for each test but on the other hand unreliable identification due to mixed infection or the specimens containing $\leq 10^{4} \mathrm{CFU} / \mathrm{ml}$. The databases currently available for both systems should be updated to enhance performance.
\end{abstract}

Keywords: Candida species, Identification, MALDI-TOF MS, Susceptibility, Urine, Vitek 2

\section{Introduction}

Because yeast in urine samples are often contaminants, confirmation of their pathogenisty should be the first step. To differentiate between contaminate and fungal infection obtaining of a second urine sample from the patient is important [1]. Clean mid-stream samples should be taken or using catheter specially in elderly [2]. Mostly candiduria patients are asymptomatic usually women and they noted when they have urinalysis or urine culture and commonly associated with vaginitis. Symptomatic patients sometimes showed cystitis, dysuria, suprapubic discomfort and when it's neglected can lead to flank pain ,pyelonephritis, oliguria , painful urination, epididiymo- orchitis , Candida prostatitis and perinephric abscess [3],[4],[5],[6]. Early identification is necessary and can be done by using Matrix Assisted Laser Desorption Ionization Time-of-Flight Mass Spectrometry (MALDI-TOF MS) which can detect directly the weight of phenotypical proteins from urine samples as yeast pellets [7]. The present study was done to identify Candida species from urine samples by MALDI-TOF MS and Vitek 2 susceptibility system in order to evaluate both methods for rapid and accurate identification of yeast spp.,. 146 positive samples for fungal infection were investigated.

\section{Materials and Methods}

\subsection{Urinalysis, Microscope and culturing}

3.370 Clean mid-stream samples were collected from patients of Hail General Hospital and Maternity Hospital in Hail ,KSA. Examination of urine samples for fungal elements by centrifugation and Gram stained, Microscopic examination were done for the presence of budding yeast cells and hyphal elements . $1 \mu \mathrm{L}$ of well -mixed urine was inoculated and spread onto SDA, Blood agar and Candida Chromogenic agar plates (BBL CHROMagar Candida, Beckton Dickinson ,France) ,for each sample using sterile plastic disposable loop (SPL Lifescience, Pocheon ,Korea). Plates were incubated in an aerobic atmosphere at $37^{\circ} \mathrm{C}$ for 18-24 hr. Growth were observed and the colonies on blood agar and SDA used for conventional methods of identification , colors on Candida Chromogenic agar were identified .

\subsection{MALDI-TOF MS Identification}

146 urine samples were Duplicated in order to use the same samples in both methods . Maldi BioTyper( Burker Daltonics, GmbH, Germany) were used and protocol by Ferreire et al [8] and Lavergne et al [9] for MALDI-TOF MS analysis were adapted , to remove leukocytes centrifugation of $5 \mathrm{ml}$ urine from each sample for 30sec were done, then the supernatant also centrifuged for $10 \mathrm{~min}$ to collect yeast cell, the sediment pellets were washed by distal water then $1.5 \mathrm{ml}$ of sodium dodecyl sulfate $0.1 \%$ was added in each tube and incubation $37^{\circ} \mathrm{C}$ for $10 \mathrm{~min}$ then centrifuged again for $10 \mathrm{~min}$ at 13,000 rpm . The supernatant was removed and the pellets were dried and suspended into adjusted volume of $10 \mu \mathrm{l} 70 \%$ formic acid and equal volume of acetonitrite and centrifuged , $1 \mu$ l of supernatant was pipetted and applied to the MALDI -TOF plates, about $1 \mu \mathrm{l}$ of matrix solution ( $\alpha$-cyano-4-hydroxycinnamic acid ) HCCA in 50\% acetonitrile and $2.5 \%$ trifluoroacetic acid (TFA) (sigma-Aldrich,France) were added to each well. 


\section{International Journal of Science and Research (IJSR) ISSN (Online): 2319-7064 \\ Index Copernicus Value (2013): 6.14 | Impact Factor (2015): 6.391}

The following adjustments were used : delay,170ns;ion source 1 voltage, $20 \mathrm{kV}$; ion source 2 voltage, $18.5 \mathrm{kV}$ ;mass range, $2-20 \mathrm{kDa}$. The resulting peaks were automatically acquired by the AutoXecute of the FlexControl v 3.3 software (Burker Daltonics, GmbH, Germany).

\subsection{Direct susceptibility testing by Vitek 2}

Yeast cell pellets were dissolved in $0.45 \%$ saline solution to prepare suspension with McFarland turbidity $1.8: 2.2$ recommended by Vitek 2 ( bioMérieux, Marcy 1'Etoile, France)[10]. Yeast suspensions were directly inoculated into Vitek 2 susceptibility testing device using AST-YS07 cards ( bioMérieux, Marcy 1'Etoile, France) .The AntiFungal Susceptibility Test (AFST) results were for Amphotericin B, Voriconazole, Fluconazole, Caspofungin and Flucytosine. MICs value were used to assign isolation susceptible(S), intermediate (I) and resistant (R) depending on Vitek 2 breaking point setting “EUCAST 2013 and CLSI 2013 D”. according to Table (1).

Table 1: Minimum Inhibitory Concentrations (MICs)

\begin{tabular}{|c|c|c|c|}
\hline Antifungal & $\mathrm{S}$ & $\mathrm{I}$ & $\mathrm{R}$ \\
\hline Amphotericin B & $\leq 1$ & - & $\geq 2$ \\
\hline *Fluconazole & $\leq 2$ & $=4$ & $\geq 8$ \\
\hline Voriconazole & $\leq 0.125$ & - & $\geq 0.25$ \\
\hline Caspofungin & $\leq 2$ & - & - \\
\hline Flucytocine & $\leq 4$ & $8 \leq$ and $\leq 16$ & $\geq 32$ \\
\hline
\end{tabular}

*Except for C.glabrata [ $\mathrm{S} \leq 1,2 \leq \mathrm{I} \leq 32, \mathrm{R} \geq 64$ ].

\section{Results}

$146(4.3 \%)$ out of 3,370 were positive samples to fungal infection depending on direct microscopic examination of urine samples, which showed yeast cells and budding . On CHROMagar different colors were present as C.albicans greenish colony, C.glabrata creamy color, C.Kursi pink color , C. dublinsiensis greenish blue ,C. lusitaniae white to mauve and C.tropicalis were metallic blue. Results showed isolates were distributed as the following 70\%C.albicans ,10\%C.glabrata ,8\%C.Kursi , 5\%C.dublinsiensis ,4\%C. lusitaniae and 3\% C.tropicalis.

\subsection{Direct MALDI-TOF MS identification results}

Species identification was (91.8\%) (134/146) of the samples The results were compared to the results of conventional method as in Table( 2).

Table 2: Candida species identification rate by using MALDI-TOF MS, $\mathrm{n}=146$

\begin{tabular}{|c|c|c|c|}
\hline species & $\begin{array}{c}\text { Total } \\
\text { number of } \\
\text { samples }\end{array}$ & $\begin{array}{c}\text { Number of } \\
\text { identified } \\
\text { samples }\end{array}$ & $\begin{array}{c}\text { Number of } \\
\text { unidentified } \\
\text { Samples * }\end{array}$ \\
\hline C.albicans & 102 & 99 & 3 \\
\hline C.galabrata & 15 & 13 & 2 \\
\hline C.kursi & 12 & 10 & 2 \\
\hline C.dublinsiensis & 7 & 5 & 2 \\
\hline C.Iusitaniae & 6 & 4 & 2 \\
\hline C.tropicalis & 4 & 3 & 1 \\
\hline Total & 146 & 134 & 12 \\
\hline
\end{tabular}

*No reliable identification insufficient peak due to urine samples mixed with bacterial infection or containing $\leq 10^{4}$
(Colony Forming Units) CFU/ml.

\subsection{Direct susceptibility testing by Vitek 2 results}

$100 \%$ agreement of species identification of the samples with conventional methods. Table (3) showing the results of antifungal susceptibility test by direct inoculation method. The duration of susceptibility testing in Vitek 2 was 23hr. C.glabrata showed resistant to Fluconazole along with Voriconazole.

Table 3: results of antifungal susceptibility testing

\begin{tabular}{|l|llll|lc|}
\hline \multirow{2}{*}{$\begin{array}{l}\text { Antifungal/species (No.of } \\
\text { isolates }\end{array}$} & \multicolumn{5}{|c|}{ Direct method } \\
\cline { 2 - 7 } & \multicolumn{3}{|c|}{ Category } & \multicolumn{2}{l|}{ MIC $(\mu \mathrm{g} / \mathrm{ml})$} \\
\cline { 2 - 7 } & $\mathrm{S}$ & $\mathrm{I}$ & $\mathrm{R}$ & $50 \%$ & $90 \%$ \\
\hline Amphotericin B & 146 & 0 & 0 & $\leq 0.25$ & 0.5 \\
\hline Fluconazole & 131 & 0 & $15^{*}$ & 2 & 4 \\
\hline Voriconazole & 132 & 0 & $14^{*}$ & $\leq 0.12$ & 0.25 \\
\hline Caspofungin & 146 & 0 & 0 & $\leq 0.25$ & $\leq 0.25$ \\
\hline Flucytocine & 146 & 0 & 0 & $\leq 1$ & $\leq 1$ \\
\hline
\end{tabular}

${ }^{*}$ C.glabrata , $\mathrm{MIC}_{50}$ and $\mathrm{MIC}_{90}$ were not calculated for single species due to low number of isolates .

\section{Discussion}

Candida is a very important pathogen, infection and dissemination can happen with serious complications .The reliability of yeast identification results in significantly improving treatment .

In the past 10 years Candida spp. are between fourth and sixth most common nosocomial infection specially with catheterization [11],[12].Previous studies have shown excellent correlation between Vitek 2 susceptibility testing, MALDI-TOF MS and conventional yeast identification [13],[14] .Based on the present work results showed higher rate of identification of specimens containing $\geq 10^{5} \mathrm{CFU} / \mathrm{ml}$ than that containing $\leq 10^{4} \mathrm{CFU} / \mathrm{ml}$ which showed insufficient peaks so considered as unreliable identification which corresponds with [15],[16].Vitek2 susceptibility testing results have been shown a reliable performance[17],[18], however Fluconazole and Voriconazole showed resistant with C.glabrata and C.kursi. The prevalence of Candida spp according to previous studies in the Kingdom of Saudi Arabia , Brazil and India[19],[20],[21] showed some variation of the percentage of each Candida spp.This study show that C.albicans $70 \%$ of the isolates followed by $10 \%$ C.galabrata, $8 \%$ C. Kursi, $5 \%$ C.dublinsiensis, $4 \%$ C. lusitaniae and 3\% C.tropicalis.

In conclusion many Candida spp ; C.albicans, C.galabrata, C. Kursi, C.dublinsiensis, C. lusitaniae and C.tropicalis have been recorded from patient admitted to Microbiology laboratory of Hail General Hospital and Hail Maternity Hospital between April 2014 and December 2015and farther investigation has to be done in different regions in the Kingdom of Saudi Arabia in order to monitor Candida spp distribution .

MALDI-TOF MS showed higher results when the specimens contain $\geq 10^{5} \mathrm{CFU} / \mathrm{ml}$ and it's fast ,easier and more economic, Vitek 2 AST-YS07 card AFST showed high

\section{Volume 5 Issue 4, April 2016




\section{International Journal of Science and Research (IJSR) \\ ISSN (Online): 2319-7064 \\ Index Copernicus Value (2013): 6.14 | Impact Factor (2015): 6.391}

performance but for Fluconazole and Voriconazole were suboptimal and subculture remain indispensable to validate the results also the databases currently available for both systems should be updated to enhance performance.

\section{Acknowledgements}

I would like to thank my sister Marwa and my father Prof. Dr.Abdallah Moursi for helping and supporting me, Microbiology Laboratory staff members in Maternity Hospital and Hail General Hospital, Hail, KSA

Declaration of interest: The authors report no conflicts of interest. The author alone is responsible for the content and writing of the paper.

\section{References}

[1] Fisher JF, Chew WH, Shadomy S, Duma RJ, Mayhall CG, House WC, "Urinary tract infection due to Candida albicans, ', Rev infected Dis, 4:1107-18,1982.

[2] Sobel JD , " Management of asymptomatic candiduria," Internat J Anti microb Agents ,11, pp.285-8,1999.

[3] Fisher JF , "Candiduria: when and how to treat it," Curr Infect Dis Rep ,2:523-30,2000.

[4] Harbach LB, Burkholder GV, Goodwin WE, "Renal candidasis .A cause of anuria," Br J Uorl , 42,pp.25864, 1970.

[5] Tennant FS, Remmers AR, Perry JE, “ Primary renal candidasis . Associated perinephric abscess and passage of fungus balls in the urine," Arch Intern Med ,122, pp.435-40,1968.

[6] Schonebeck J, " Studies on Candida infection of the urinary tract and on the antimycotic drug 5fluorocytosine," Scand J Urol Nephrol 11, pp.748,1972 .

[7] Stevenson LG , Darke SK , Shea YR , Zelazny AM, Murray PR , " Evaluation of matrix assisted laser desorption ionizing -time of filght mass spectrometry for identification od clinically important yeast species," J Clin Microbiol , 48, pp. 3482-3486,2010.

[8] Ferreira L, Sánchez-Juanes F , González-Avila M , Cembrero -Fucînos D , Herrero -Hernándaz A , González - Buitrago JM , et al “ Direct identification of urinary tract pathogens from urine samples by matrix laser desorption ionizing- time of flight mass spectrometry ," J Clin Microbiol , 48 , pp .2110-5,2010.

[9] Lavergne RA , Chauvin P , Valentin A , Fillaux J ,Roques- Malecaze C , Arnaud S, etal , “An extraction method of positive blood culture for direct identification of Candidia species by matrix -assisted laser desorption ionizing time of flight mass spectrometry," Med Mycol, 51 , pp. 652-6,2013.

[10] BioMérieux SA VITEK 2 System product information,410791, Mercy-I'Etoile,France, 2010.

[11] Bouza E, Munoz P , " Epidemiology of Candidemia in intensive care units ," Int Antimicrob Agents , 32, 2, pp.S87-S91, 2008.

[12] Sobel DJ , " Controversies in the diagnosis of Candiduria : what is the critical colony count ?," Curr Treatment Opinions Infect Dis , 4, pp.81-3,2002.
[13] Won EJ , Shin JH , Lee k , Kim MN , Lee HS, Park YJ,etal " Accuracy of species level identification of yeast isolates from blood cultures from 10 university hospitals in South Korea by use of the matrix -assisted laser desorption ionizing-time of flight mass spectrometry-based Vitek MS system," J Clin Microbiol,51,pp. 3063-5,2013.

[14] Westblade LF , Jennemann R , Branda JA , Bythrow M , Ferraro MJ , Garner OB, etal, “ Multicenters studies evaluating the Vitek MS system for identification of medically important yeast," J Clin Microbiol , 51, pp. 2267-72,2013.

[15] Dhiman N , Hall L , Wohlfiel SL , Buckwalter SP , Wengenack NL , " Performance and cost analysis of matrix -assisted laser desorption ionization-time of flight mass spectrometry for routine identification of yeast ," J Clin Microbiol , 49 , pp.1614-1616, 2011.

[16] Schmalreck AF,Lacner M, Becker K, Fegeler W, Czaika V,etal, “ Phylogenetic relationships matter: antifungal susceptibility among clinically relevant yeast," Antimicrob Agent Chemother,58,pp. 1575$1585,2014$.

[17] Pfaller MA , Diekema DJ , Procop GW , Rinaldi MG, "Multicenters comparison of VITEK2 antifungal susceptibility test with the CLSI broth microdilution reference method for testing amphotericinB ,flucytocine ,and voriconazole against Candida spp.," J Clin Microbiol ,45,pp.3522-3528,2007.

[18] Pfaller MA , Diekema DJ , Procop GW , Rinaldi MG, "Multicenter comparison of VITEK2 antifungal susceptibility test with the CLSI broth microdilution reference method for testing fluconazole against Candida spp.” J Clin Microbiol , 45, pp. 796-802,2007.

[19] Al-Tawfiq AJ ,Abed MS, “ Prevalence and antimicrobial resistance of health care associated bloodstream infections at a general hospital in Saudi Arabia," Saudi Med J, 30 , pp.1213-128,2009.

[20] Chaves GM , Diniz MG , Da Silva-Roch WP , De Souza LB , Gondim LA , Ferrerie MA, et al ,"Species distribution and virulence factors of Candida spp. Isolated from oral cavity of Kidney transplant recipients in Brazil,” Mycopathologia, 175, pp.255-263 2013.

[21] Mohandas V , Balla M, " Distribution of Candida species in different clinical samples and their virulence: biofilm formation, protienase and phospholipase production :a study on hospitalized patients in southern India ," J Glob Infect Dis ,3 , pp.4-8,2011.

\section{Author Profile}

Soha Abdallah Moursi received the B.S. and M.S. degrees in Vet., Medical Sciences , Microbiology Mycology 2002,2005 ,Zagazig University Egypt . During2006-2010,received her PhD, she stayed in Berlin Germany for the scholarship in Humboldt Universität, Konsiliarlabor für Dermatophyten, Institut für Mikrobiologie und Hygiene (Charité-Universtätmedidzin) to study and finish the practical of her $\mathrm{PhD}$, under supervision of $\mathrm{Dr}$. Yvonne Gräser and Prof Dr. Mohammed Taha ,2011 working as Assistant Professor in Dept., of Clinical laboratory Science , Applied Medical science College ,Hail University ,KSA 